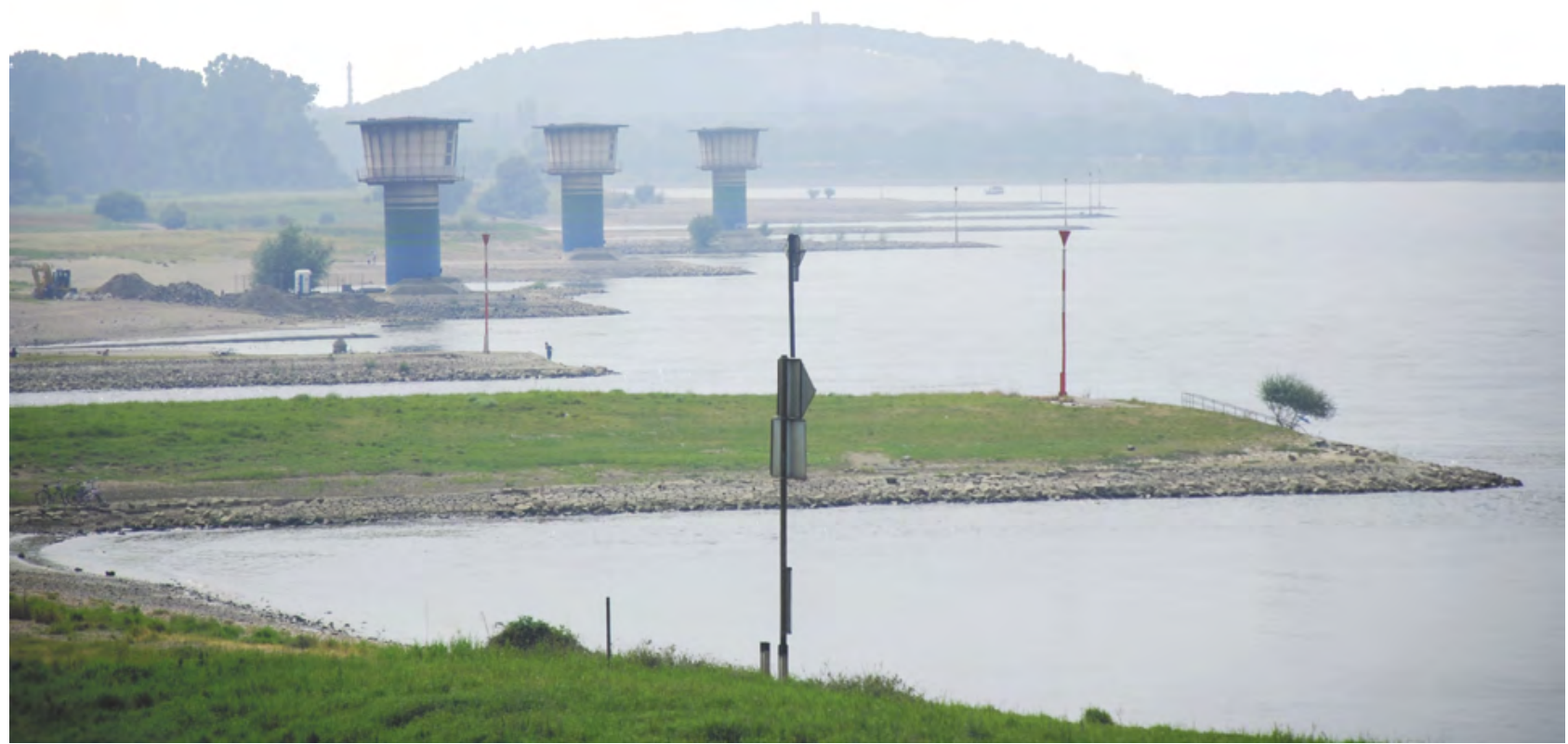

Faculté d'architecture, d'ingénierie architecturale, d'urbanisme de l'Université catholique de Louvain 


\title{
Investigations territoriales et spéculations architecturales
}

\author{
Olivier Bourez et Guillaume Vanneste
}

Comment enseigner le projet d'architecture? Et comment la conduite de cet enseignement s'appuie sur les ressorts internes de la démarche projectuelle, pour informer l'étudiant à la fois sur le comment et le pourquoi du projet d'architecture.

L'atelier d'architecture cherche à éveiller chez l'étudiant la compréhension des ressorts multiples et disponibles au développement du projet d'architecture aux moyens de certains outils de recherche que nous présenterons à travers deux dispositifs : investigations territoriales et spéculations architecturales.

\section{Dessiner pour voir}

Pour faire connaissance avec un territoire, c'est le dessin qui nous sert d'introduction. Le dessin - descriptif - comme un regard sur l'inconnu, découvre, scrute et cherche. Cherche à comprendre. Différents regards, comme différents thèmes, peuvent être portés sur ce que l'on ne connaît pas.

On peut faire plusieurs dessins d'une même chose. On peut faire plusieurs fois le même dessin. On peut aussi faire le même dessin de plusieurs choses différentes entre elles. Chaque fois, le dessin nous enseigne, il découvre des géométries, des profils, des rythmes. II regarde attentivement comme pour imprimer les traits - le portrait - d'un visage dans sa mémoire. C'est bien de cela qu'il s'agit. Au travers du dessin - des dessins -, on cherche à voir les formes de la ville, les raisons du territoire. II s'agira de comprendre essentiellement les éléments physiques qui structurent, organisent, partagent et qualifient le territoire. À regarder de près un territoire, attentivement, sans une arrière-pensée, l'on produit essentiellement du savoir. Faire connaissance. Se présenter. Représenter.

Ici, il s'agirait avant tout de produire de la connaissance (co-naître, faire naître ensemble savoir et projet) dans une visée transformative, tournée vers une temporalité au-devant de nous, en spéculant sur des hypothèses de mutation qui permettront d'entrevoir le territoire autrement qu'une réalité intangible et figée, de le comprendre comme une construction en devenir, comme un substrat à sa propre transformation, un palimpseste sans cesse amendé, un potentiel de projet.

Un territoire, une ville. Nos projets s'installent dans des lieux et des espaces. Le projet est donc une occasion de lecture de ces lieux. Une écriture aussi. En atelier, le projet consiste à concevoir et représenter un ou plusieurs édi- fices dans l'un de ces lieux. II nécessite en même temps qu'il la construit, une connaissance du territoire. L'architecture devrait s'en trouver enrichie et, par réciprocité, le territoire gagnera à son accueil. La ville n'est pas que la feuille ou se pose le dessin, elle en fait partie et les traits de sa composition s'équilibrent, se frôlent ou se contrastent, les textures de son tissu s'ouvrent ou se resserrent.

\section{Investigations territoriales}

L'office de l'architecte est dès lors plein de questionnements. Qu'est-ce qui fait la spécificité de cette ville ou ce morceau de paysage ? Qu'est-ce qui fait que ce site est celui-là et pas un autre ? Qu'est-ce qui se trame ici et qui ne se trame nulle part ailleurs?

L'analyse est une lecture. Propre au regard du lecteur, elle est un prisme qui donne à voir une réalité choisie. Elle n'est donc pas unique et sa richesse réside, entre autres, dans le caractère pluriel et dans la sélection effectuée au travers de ce travail d'investigation.

L'analyse, analusis, résolution d'un tout en ses parties, est un mode d'investigation, une lecture des unicités. Elle traduit les questions générales dans la langue architecturale. À partir de l'information des cartes de géographes, l'analyse dessinée investira les dimensions physiques qui produisent la spécificité des territoires : le relief, l'eau, la végétation, les tissus urbains ou les typologies construites, les traces historiques, les infrastructures... L'analyse dégagera les éléments les plus déterminants, les relations qui les structurent mutuellement et les échelles qui les voient se produire: le bâti suit-il la vallée ? Le relief génèret-il des infrastructures qui se répètent? À quelle échelle ? La typologie d'habitat est-elle héritée de pratiques historiques située dans ce lieu?

Nous tenons en effet que l'unicité de la situation n'est pas magique ou abstraite, elle s'écrit simplement dans des élé- 
ments physiques et dans les rapports qu'ils entretiennent (la rationalité minimale de Secchi Viganò).

L'analyse aide à la constitution d'un fond de plan (synthèse de la situation existante) sur lequel les hypothèses nouvelles seront jetées. C'est au travers de ce que l'analyse aura révélé qu'un projet rejoint la situation spécifique donnée et qu'il participe d'une histoire structurelle qui dépasse son sort individuel. C'est parce qu'on s'est intéressé aux autres composants du lieu que l'édifice peut être plus qu'un objet isolé. S'inscrire au compte des édifications suppose qu'on se soit préoccupé de ce qui était déjà écrit.

L'analyse est un projet. Une analyse isolée pourrait s'avérer épuisante parce que précisément inépuisable. C'est pourquoi le projet s'engagera simultanément à l'analyse de manière à produire des orientations de travail se nourrissant dans la réciprocité de chacun. Pour l'énoncer autrement le projet se situe à la croisée de l'histoire et de son devenir. Le travail du projet au présent consiste à présager de l'avenir dans une situation héritée du passé. Aussi analyser et projeter sont les deux faces d'une même pièce, irréductibles l'une à l'autre mais néanmoins inséparables.

Les étudiants entament leur travail en groupe et entreprennent des investigations territoriales. Au travers du dessin, de visites de site et de recherches thématiques, ils convoquent les éléments du territoire qui vont construire les fondements d'une lecture spécifique, analytique. Les limites d'investigations sont a priori inconnues et multiples en fonction des sujets investis. C'est précisément le travail qui permet de les trouver, si tant est qu'il y en ait. La structure est l'ensemble des éléments et leurs rapports.

Considérons que l'analyse dessinée d'une ville et de son territoire connaît deux temps majeurs : la lecture des éléments du territoire et la mise en évidence des rapports.

L'alternance proposée par l'atelier, tantôt une ville constituée, tantôt un territoire plus vaste, cherche à caractériser, voire caricaturer ces lectures et ces mises en rapports pour les rendre lisibles à l'étudiant.
D'un côté, on peut analyser le territoire avec des outils semblables à ceux de l'analyse urbaine : si la ville se comprend à travers l'articulation de 3 éléments formant le tissu urbain (le parcellaire, le bâti, la voirie), le territoire peut s'appréhender à travers l'articulation de 3 éléments de même nature : les découpages (limites territoriales, institutionnelles, géographiques...), I'urbanisation (villes, villages, routes-rubans...), les réseaux (routes, chemins de fer, hydrographie, mais aussi réseaux d'électricité, collecte des eaux usées.... $)^{1}$.

De l'autre, I'analyse du territoire se différencie de l'analyse urbaine par l'usage des échelles : si on peut visualiser l'articulation entre le bâti, la voirie et le parcellaire à une seule échelle (Fortier 1989), pour appréhender un territoire, i faut accumuler les échelles d'analyse et inventer les modes de visualisation des relations entre elles ${ }^{2}$.

Enfin, tout territoire (urbain ou autre) s'appréhende en comprenant l'accumulation des couches, les superpositions, juxtapositions ou effacements de son histoire : le palimpseste ${ }^{3}$. Dans un premier temps de préparation, il s'agît de faire un tri dans les informations d'une carte géographique ou cadastrale pour donner à voir certaines réalités. Dans un second, on vise à mettre en évidence des rapports. II s'agit d'interprétations sur la base constative qui peuvent être influencées par ce que les visites et les dessins auront révélé. Les rapports sont spécifiques au territoire. Ils explorent I'hydrographie et la topographie, le tissu et la structure des vides, les réseaux et leur connectivité, la chronologie et la typo-morphologie...

L'ensemble des dessins doit aider à dégager le plus significatif, le plus prenant le plus décisif pour parler de la structure. On pourrait dire aussi qu'on cherche la différence dans laquelle s'établit cette ville ou ce territoire.

L'ordre sous-jacent dégagé n'est pas un pur jeu formel ou de composition. Le travail cherchera à découvrir et à relever le plus de déterminants possibles pour comprendre la fabrication du seu morceau de territoire. Si ce morceau de territoire est unique à certains égards, avec d'autres il partage sans doute des éléments de comparaison. 


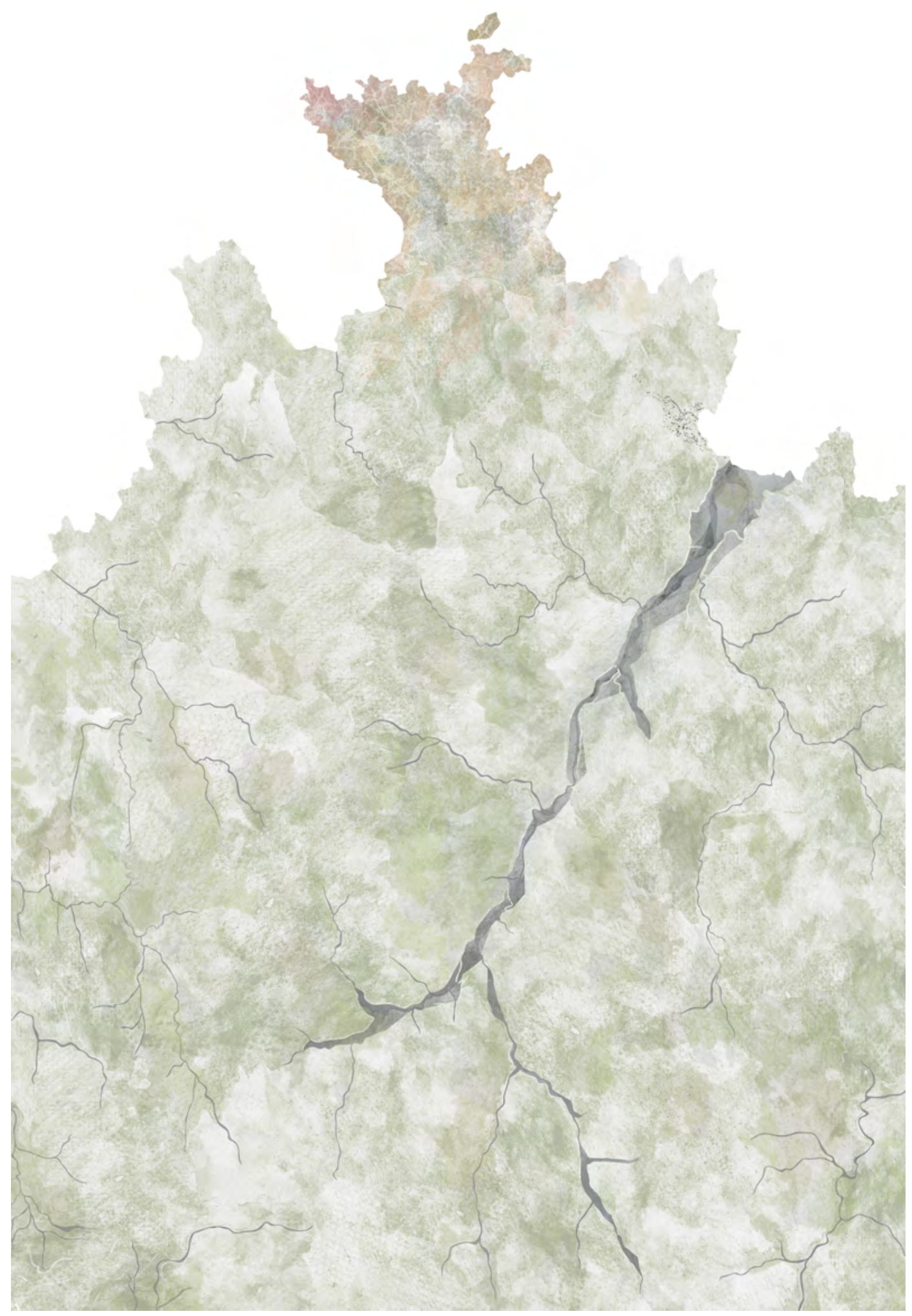


L'analyse et la compréhension du territoire sont, a priori, le but ultime des investigations territoriales. Ce dessin se veut dès lors non seulement rétroactif mais également proactif puisqu'il s'agit d'y spéculer sur des hypothèses d'implantation et de construction. Par ailleurs, il s'agira, grâce à ce travail, de supposer des hypothèses architecturales qui prennent sens et valeur aux différentes échelles, aux différentes thématiques investies.

\section{Spéculation architecturale}

C'est autour de l'édifice construit, de sa mesure et de son programme que se déploient les questions de l'étudiant qui cherche, au moyen d'arguments, à trouver et communiquer les raisons d'un projet. L'édifice étant l'objet du travail de l'étudiant, qui évolue bel et bien au sein de sa formation d'architecte, il est légitime d'organiser et structurer le projet, sa représentation et son argumentation autour de l'espace architecturé. Le recours aux éléments urbains, programmatiques, aux standards, à tout ce qui pourrait être nommé une "contrainte" au sens de Stravinsky ${ }^{4}$, servent à guider les spéculations architecturales. Idéalement, ce travail spéculatif n'est pas absolument successif aux investigations : de même qu'il navigue entre les échelles, les références et les dessins, l'étudiant doit faire appel à ces deux démarches de manière intégrée et connexe plutôt que linéaire et successive. La maturité de l'étudiant et son expérience acquise articulent spéculation et investigation et les transforment en projet.

Plusieurs échelles habitent le projet. Autonomes et complémentaires, elles n'ont pas de compte à rendre à un quelconque processus linéaire qui tiendrait lié ensemble, d'une part le début de la lecture avec les grandes échelles et l'amplitude territoriale, d'autre part la fin de l'histoire ou la mise en œuvre du projet d'édifice et l'échelle de ce dernier. Au contraire. À tout moment, les multiples échelles des dessins se parlent, se complètent et se contredisent. Les narrations du projet peuvent ne pas être chronologiques. Ellipse, retour en avant, parallaxe. Elles alimentent un feu constant de réflexions où le cumul des échelles est autant de remises en question ou de vérifications et de confirmations d'une hypothèse. Cette mobilité n'est pas (toujours) immédiate, elle s'apprend et se cultive, pour finir par se sentir confortable sur ses sentiers non balisés.

Plusieurs projets habitent le même dessin. La référence nous parle de ces similitudes de formes que l'on peut retrouver d'un projet à l'autre. Comme des surimpressions photographiques, la référence évoque les rémanences dans l'histoire de l'architecture ou des formes en général. Ces ressemblances et ces analogies sont inspirantes pour comprendre et manipuler les dispositifs formels et les types que l'on met en place au sein de son propre projet. Elles sont autant de mélodies qui font écho à celle qui se joue dans le projet en cours de fabrication.

Les spéculations architecturales se construisent par la manipulation des multiples échelles de dessins et des multiples précédents des formes et des compositions que l'on convoque dans le projet. Elles sont autant de mécanismes de projet suggérés pour mettre le futur en forme.

Au travers des investigations et des spéculations, la recherche produite par l'étudiant, laisse à voir les enjeux du projet en sa qualité d'objet même de la recherche mais aussi d'outil de recherche. L'étudiant construit à la fois le projet comme un objet architecturé, conjointement, il instruit le projet du territoire dans lequel il s'implante et, enfin, il acquiert la connaissance d'une démarche, d'un savoir-faire, d'un processus de construction mentale et dessinée du projet. En ce sens, l'atelier se construit sur la nature double de ce qui fait projet, à la fois lecture et écriture, description et transformation. 